\title{
Pacific
}

Journal of

Mathematics

\section{ON THE SHAPE OF FUNDAMENTAL DOMAINS IN}

$$
\operatorname{GL}(n, \mathbf{R}) / \mathbf{O}(n)
$$

\author{
DOUGLAS MARTIN GRENIER
}




\title{
ON THE SHAPE OF FUNDAMENTAL DOMAINS \\ IN $\mathrm{GL}(n, \mathbf{R}) / \mathrm{O}(n)$
}

\section{Douglas Grenier}

\begin{abstract}
We investigate parameters for the symmetric space $H=G / K$, $G=\mathrm{GL}(n, \mathbf{R}), K=\mathrm{O}(n)$, in the sense of positive definite quadratic forms. This leads to a description for the fundamental domain $H / \Gamma$ where $\Gamma$ is an arithmetic subgroup of $G$. We also see interesting relations with the Siegel sets. This enables us to explicitly describe Satake compactifcations of $H / \Gamma$. We will also consider the behavior at the "bottom" of the fundamental domains.
\end{abstract}

1. Introduction. The problem of reduction of quadratic forms is an old one. When the subject is positive definite quadratic forms, the first definition of reduction was achieved by Hermite [8]. However, it is Minkowski's reduction that is the most familiar, primarily because when we view the quadratic forms geometrically, Minkowski's reduction domain is easily seen to have a finite number of boundary components while this was not known for Hermite's. One may consult Cassels [3] or Terras [14] for more of the historical details and for a definition of these domains.

In modern language, positive definite quadratic forms may be considered as a symmetric space. Denote the space of positive quadratic forms by $\mathscr{P}_{n}$, and let $G=\mathrm{GL}(n, \mathbf{R}), K=\mathrm{O}(n)$. Then $\mathscr{P}_{n}$ may be identified with $G / K$ by:

$$
g K \mapsto^{T} g g
$$

for any $g \in G$. We will be most interested in the space of quadratic forms of determinant one which will be denoted $\mathscr{S P P}_{n}$. This may be obtained from $G / K$ by modding out by the center of $G$. If $\Gamma_{n}$ stands for $\mathrm{GL}(n, \mathbf{Z}) /\{ \pm I\}, \Gamma_{n}$ acts discontinuously on $\mathscr{S P P}_{n}$ by:

$$
Z \mapsto Z[\gamma]={ }^{T} \gamma Z \gamma
$$

where $Z \in \mathscr{S P}_{n}$ and $\gamma \in \Gamma_{n}$. We will always use this notation $Z[X]={ }^{T} X Z X$ where $X \in \mathbf{R}^{n \times k}$ for any $k$ (including $k=1$ so that $X$ is a vector). Then a fundamental domain in $\mathscr{S P}_{n}$ under the action of $\Gamma_{n}$ is a subset of $\mathscr{S P P}_{n}$ which may be identified with the quotient space $\mathscr{S P P}_{n} / \Gamma_{n}$ and which represents the reduced forms. For 
studying arithmetic subgroups of $\mathrm{GL}(n, \mathbf{R})$ and automorphic forms an Hermite style fundamenal domain was preferable to Minkowski's. In [5] such a fundamental domain was defined, and it was shown that it was indeed possible to explicitly determine the (bottom) boundary components. This is also in [6].

The object of this paper is to develop in more depth various properties of the fundamental domains of [5] and [6]. We will explicitly determine compactifications of these domains and hence the quotient groups. These will be Satake compactifications of $\mathscr{S P}_{n} / \Gamma_{n}$ modelled after those originally considered by Satake [12] for the Siegel space and generalized by Satake and others (see, for example, $[13,1,16])$ to many algebraic groups. We will describe the topologies of certain compactifications of the fundamental domains and the quotient groups, and see the relation between the so-called boundary components and the parabolic subgroups of $G$, as in the compactifications of Satake and Baily-Borel. We believe that these compactifications will be quite useful in the study of automorphic forms for $\operatorname{GL}(n, \mathbf{R})$, specifically towards generalizing the results of [7] that defined a map sending automorphic forms for $\mathrm{GL}(n, \mathbf{R})$ to forms for $\mathrm{GL}(n-1, \mathbf{R})$. The nature of the Satake topology defined in $\S 4$ indicates that there should be similar maps relative to each parabolic subgroup, sending an automorphic form for $\mathrm{GL}(n, \mathbf{R})$ to a product of lower rank automorphic forms. This in turn might lead to explicit Maass-Selberg relations for $\operatorname{GL}(n, \mathbf{R})$. The Siegel sets play an important role both in the definition of the fundamental domain and the compactification. The relation of the fundamental domain to the Siegel sets also has a bearing on many problems in automorphic forms and harmonic analysis on $\mathrm{GL}(n, \mathbf{R})$, for example as in Huntley [9] (also see [14]). Mostly we concentrate on the action of $\mathrm{GL}(n, \mathbf{Z})$ on $\mathscr{S P P} n$, but since an arithmetric subgroup of $G$ is commensurable with $G L(n, Z)$, these ideas are easily extended to fundamental domains for arbitrary arithmetic subgroups. Due to the celebrated arithmeticity theorems of Margulis (summarized in [15]), this last notion may deserve more attention than it is given here.

In the following, in $\S 2$ we will briefly summarize the results of [5, 6] concerning the fundamental domain we will consider here. Then these results will be modified and/or extended to suit the purposes of this paper. Section 3 will take a closer look at the Siegel sets (which are already preset in the definition of the fundamental domain). We will investigate the relations between the fundamental domain and 
the Siegel sets, including how they are used to establish a reduction algorithm, and in work of Huntley on the spectrum of the Laplacian. In $\S 4$ the fundamental domain will be compactified in the style of Satake [12]. This also gives a compactification of the quotient space $\mathscr{S P}_{n} / \Gamma_{n}$. The author would like to thank S. Zucker for several helpful discussions on these last matters.

2. Fundamental domains in $\mathscr{S P}_{n}$. This section is partly a summary of results from $[5,6]$, although we have made various modifications and additions. Throughout we will use the following coordinates on $\mathscr{S P P}_{n}$ :

For any $Z \in \mathscr{S P P}_{n}$, let

$$
Z=\left(\begin{array}{cc}
y^{-1} & O \\
O & y^{1 /(n-1)} Z^{\prime}
\end{array}\right)\left[\begin{array}{cc}
1 & T \\
0 & I
\end{array}\right]
$$

where the square bracket notation is as before, and $y>0, Z^{\prime} \in$ $\mathscr{S P P}_{n-1}, x \in \mathbf{R}^{n-1}$. These may appear a little strange at first, but they have been chosen to point out parallels with the upper half-plane $H$ (where $x$ and $y$ would just be the real and imaginary parts of $z \in H$ ), and so that $|Z|^{\prime}=1$, which enables us to define the coordinates recursively. More explicitly the identification of $\mathscr{S P}_{2}$ with $H$ is given by:

$$
\begin{gathered}
H \rightarrow \mathscr{S P P}_{2}, \\
x+i y=z \mapsto\left(\begin{array}{cc}
y^{-1} & 0 \\
0 & y
\end{array}\right)\left[\begin{array}{ll}
1 & x \\
0 & 1
\end{array}\right] .
\end{gathered}
$$

The coordinates defined in (1) are called partial Iwasawa coordinates since if we repeat this decomposition for $Z^{\prime}$, and so on, we get the Iwasawa decomposition for $Z \in \mathscr{S P}_{n}$, which it is convenient to write

(2) $Z=y^{-1}\left(\begin{array}{lllll}1 & & & & \\ & y_{1}^{2} & & & \\ & & \left(y_{1} y_{2}\right)^{2} & & \\ & & \ddots & \\ & & & & \left(y_{1} y_{2} \ldots y_{n-1}\right)^{2}\end{array}\right)\left[\begin{array}{ccc}1 & x_{i j} & \\ & \ddots & \\ & & 1\end{array}\right]$.

Note that the $y_{i}$ correspond to the simple roots of the Lie algebra of $G$, which in the Lie group setting are actually $y_{i}^{-2}$ for $i=$ $1,2, \ldots, n-1$. The partial Iwasawa coordinates demonstrate an embedding of the lower rank symmetric spaces $\mathscr{S P}_{m}$ into $\mathscr{S P P}_{n}$. 
Using the partial Iwasawa decomposition (1) we can now define a fundamental domain for $\Gamma_{n}$ in $\mathscr{S P P}_{n}$, corresponding to the quotient space $\mathscr{S P P}_{n} / \Gamma_{n}$. Let $\mathscr{F}_{n}$ be the set of all $Z \in \mathscr{S P}_{n}$ satisfying:

(D1) For all $\left(\begin{array}{cc}a^{T} b \\ c & D\end{array}\right) \in \Gamma_{n},\left(a+{ }^{T} x c\right)^{2}+y^{n /(n-1)} Z^{\prime}[c] \geq 1$ where $a \in \mathbf{Z}, b, c \in \mathbf{Z}^{n-1}$, and $D \in \mathbf{Z}^{n-1 \times n-1}$.

(D2) $Z^{\prime} \in \mathscr{F}_{n-1}$.

(D3) $0 \leq x_{12} \leq 1 / 2,\left|x_{1 j}\right| \leq 1 / 2$ for $j=3, \ldots, n-1$.

This is slightly different from the definition in $[5,6]$, as there the approach was more in keeping with the idea of quadratic forms (and not necessarily just those of determinant one). Here, we want to see a cusp as $y \rightarrow \infty$. As indicated in the introduction, this fundamental domain has the advantage of being defined recursively as in Hermite's original idea, but it was shown in $[5,6]$ that only a finite number of combinations of $a \in \mathbf{Z}$ and $c \in \mathbf{Z}^{n-1}$ are necessary in condition (D1).

Since $\mathscr{S P}_{2}$ is identified with the upper half-plane $H$, the fundamental domain above for $n=2$ is just half of the usual one in $H$, namely $\mathscr{F}_{2}$ is identified with $D \subset H$ where

$$
D=\left\{x+i y=z \in H \mid x^{2}+y^{2} \geq 1,0 \leq x \leq 1 / 2\right\} .
$$

$\mathscr{F}_{2}$ is compactified by adding the point at infinity, i.e., $\mathscr{F}_{2}{ }^{*}=\mathscr{F}_{2} \cup\{\infty\}$ is a compact subset of $H^{*}=H \cup\{\infty\}$ corresponding to $H^{*} / \Gamma_{2}$. To see how to compactify $\mathscr{F}_{n}$ when $n>2$ we will first consider the example of $\mathscr{F}_{3}$. This fundamental domain was pictured in [4]. For $n=3$ the Iwasawa decomposition (2) becomes:

$$
Z=y^{-1}\left(\begin{array}{ccc}
1 & & \\
& y_{1}^{2} & \\
& & \left(y_{1} y_{2}\right)^{2}
\end{array}\right)\left[\begin{array}{ccc}
1 & x_{12} & x_{13} \\
& 1 & x_{23} \\
& & 1
\end{array}\right] \text {. }
$$

The fundamental domain $\mathscr{F}_{3}$ can be given as the set of all $Z \in \mathscr{S P}_{3}$ satisfying the following inequalities:

(i) $x_{12}^{2}+y_{1}^{2} \geq 1$,

(ii) $x_{12}^{2}+y_{1}^{2}\left(x_{23}^{2}+y_{2}^{2}\right) \geq 1$,

(iii) $\left(x_{12}-x_{13}\right)^{2}+y_{1}^{2}\left(\left(1-x_{23}\right)^{2}+y_{2}^{2}\right) \geq 1$,

(iv) $\left(1-x_{12}+x_{13}\right)^{2}+y_{1}^{2}\left(\left(1-x_{23}\right)^{2}+y_{2}^{2}\right) \geq 1$,

(v) $x_{23}^{2}+y_{2}^{2} \geq 1$

(vi) $0 \leq x_{12} \leq 1 / 2$,

(vii) $0 \leq x_{23} \leq 1 / 2$,

(viii) $\left|x_{13}\right| \leq 1 / 2$.

Inequalities (i)-(iv) come from condition (D1) in the definition, while (v) and (vii) come from (D2). For $y_{1} \geq M$, for some sufficiently 
large $M$, the first four inequalities above are unnecessary to have $Z \in \mathscr{F}_{3}$. Thus we might say that as $y_{1} \rightarrow \infty$ what was the point at infinity for $n=2$ now becomes $\mathscr{F}_{2}$ for $n=3$. As $y_{2} \rightarrow \infty$ we get similar behavior except that it is inequalities (ii) $-(\mathrm{v})$ that become unnecessary. However, we still see $\mathscr{F}_{2}$ as we approach the cusp determined by $y_{2} \rightarrow \infty$.

To see how this would carry over to the general situation it will be convenient to write $Z \in \mathscr{F}_{n}$ as:

$$
Z=y^{-1}\left(\begin{array}{cc}
1 & O \\
O & Z_{1}
\end{array}\right)\left[\begin{array}{cc}
1 & T \\
0 & I
\end{array}\right]
$$

so $Z_{1}=y^{(n / n-1)} Z^{\prime}$ from the partial Iwasawa decomposition (1). Let us define $a_{1}(Z)$ to be the upper left corner entry of the matrix $Z \in \mathscr{P}_{n}$. Then

$$
a_{1}(Z[\gamma])=Z\left[\begin{array}{l}
a \\
c
\end{array}\right]
$$

where $\gamma=\left(\begin{array}{cc}a & \\ c & b\end{array}\right)$ as usual. A simple computation gives

$$
y Z\left[\begin{array}{l}
a \\
c
\end{array}\right]=\left(a+{ }^{T} x c\right)^{2}+Z_{1}[c]
$$

If $c=\left(\begin{array}{c}a^{\prime} \\ c^{\prime}\end{array}\right)$, with $c^{\prime} \in \mathbf{Z}^{n-2}$, then we can also decompose $Z^{\prime}$ as we did for $\mathbf{Z}$ above and compute $a_{1}\left(Z^{\prime}\right)$. Thus

$$
y^{\prime} Z^{\prime}[c]=\left(a^{\prime}+{ }^{T} x^{\prime} c^{\prime}\right)^{2}+Z_{2}\left[c^{\prime}\right]
$$

where $x^{\prime}, y^{\prime}$ and $Z_{2}$ would correspond to $x, y$ and $Z_{1}$ for $Z$. We also know $y^{\prime} Z^{\prime}=y_{1}^{-2} Z_{1}$, so we can rewrite the above equation as:

$$
y_{1}^{-2} Z_{1}[c]=\left(a^{\prime}+{ }^{T} x^{\prime} c^{\prime}\right)^{2}+Z_{2}\left[c^{\prime}\right]
$$

If we repeat the arguments above we eventually arrive at the following:

LEMMA 1. $Z_{i}[c] \geq y_{i}^{2}$ for all $c \in \mathbf{Z}^{n-i}-\{0\}$.

Since for each $i, y_{i} \rightarrow \infty \Rightarrow y \rightarrow \infty$ (so $\left.y^{-1} \rightarrow 0\right)$, as $y_{i} \rightarrow \infty, \mathbf{Z}$ breaks into two blocks of sizes $i$ and $n-i$, the first of which goes to 0 , the second to $\infty$. We will write:

$$
\text { As } y_{i} \rightarrow \infty, \mathscr{F}_{n} \rightarrow \mathscr{F}_{i} \times \mathscr{F}_{n-i} \text {. }
$$

A variation of Lemma 1 will also be useful:

LEMMA 2. $Z_{1}[c] \geq y_{1}^{2} \cdots y_{k}^{2}$ for all $c \in \mathbf{Z}^{n-1}$ which have $c_{j} \neq 0$ for all $j \geq k$.

This may be proved by applying equation (4) and Lemma 1. 
3. Siegel sets in $\mathscr{S P P}_{n}$. In this section it will sometimes be convenient to consider the region $\mathscr{F}_{n}^{\prime}=\bigcup_{\gamma \in D_{n}} \mathscr{F}_{n}[\gamma]$ where $D_{n}$ is the subgroup of diagonal matrices

$$
\left(\begin{array}{lll} 
\pm 1 & & \\
& \ddots & \\
& & \pm 1
\end{array}\right) .
$$

We will denote this fundamental domain $\mathscr{F}_{n}^{\prime}$. Condition (D3) would become for $\mathscr{F}_{n}^{\prime}$ :

(D3') $\left|x_{i}\right| \leq 1 / 2$ for all $i$.

while (D2) would become accordingly:

(D2') $Z^{\prime} \in \mathscr{F}_{n-1}^{\prime}$,

with (D1) remaining the same. Then $\mathscr{F}_{n}^{\prime}$ is symmetric about $x_{i j}=0$ for all $i, j$ with $1 \leq i<j \leq n$.

The Iwasawa decomposition of $G$ is written $G=K A N$, where $K=\mathrm{O}(n), A$ is the diagonal subgroup of $G$, and $N$ is the nilpotent subgroup of upper triangular matrices with 1 on the diagonal. A Siegel set in $G$ is a subset of the form $K \cdot A_{t} \cdot w$ where $A_{t}=\{a \in A \mid \alpha(a) \leq t\}$ for all simple roots $\alpha$ and $w$ is a compact subgroup of $N$ containing a neighborhood of $I$. More details may be found in [2].

Analogously, we may define Siegel sets for $\mathscr{S P}_{n}$. Since we have seen that the $y_{i}$ in the decomposition (2) correspond to the simple roots of the Lie algebra of $G$ and under the map $g \mapsto^{T} g g, g=k a n$ is sent to $a^{2}[n]$, define the Siegel set $\mathscr{S}_{t, 1 / 2}$ by

$$
\mathscr{S}_{t, 1 / 2}=\left\{Z \in \mathscr{S P P}_{n}\left|y_{i} \geq t^{-1 / 2},\right| x_{i j} \mid \leq 1 / 2\right\} \text {. }
$$

Then we have the following:

THEOREM 1. $\mathscr{S}_{1,1 / 2} \subset \mathscr{F}_{n}^{\prime} \subset \mathscr{S}_{4 / 3,1 / 2}$.

Proof. Clearly this is true for $n=2$. One need only consider the standard picture of $\mathscr{F}_{2}^{\prime}$ in the upper half-plane $H$. Using the partial Iwasawa decomposition (3), condition (D1) of the definition of $\mathscr{F}_{n}$ becomes:

$\left(\mathrm{D} 1^{*}\right)\left(a+{ }^{T} x c\right)^{2}+Z_{1}[c] \geq 1$

for all $a, c$ forming the first column of $\gamma \in \Gamma_{n}$. If we choose $a=0$, $c=e_{1}$, the first standard unit vector in $\mathbf{R}^{n-1}$ we get $x_{1}^{2}+y_{1}^{2} \geq 1$ and since $\left|x_{1}\right| \leq 1 / 2, y_{1} \geq 3 / 4$. This argument can be applied in turn to $Z^{\prime} \in \mathscr{F}_{n-1}^{\prime}$ to get $y_{2} \geq 3 / 4$ and so on. Thus we have the second inclusion. To get the first we need to show that if $y_{i} \geq 1$ for all $i$, and $\left|x_{i j}\right| \leq 1 / 2$ for all $i, j$, then $Z \in \mathscr{F}_{n}^{\prime}$. As mentioned above, we know 
this for $n=2$, so we proceed by induction. Since $Z_{1}$ is positive, if $\lambda$ is the least eigenvalue of $Z_{1}$, then $Z_{1}[c] \geq \lambda I[c]$. The eigenvalues of $Z_{1}$ are $y_{1}^{2},\left(y_{1} y_{2}\right)^{2}$, etc., so $\lambda \geq 1$. If $c \neq 0, Z_{1}[c] \geq \lambda \geq 1$ so condition $\left(\mathrm{D} 1^{*}\right)$ is satisfied. If $c=0$ then $a$ must be \pm 1 to have $\gamma \in \Gamma_{n}$ which again makes $\left(\mathrm{D} 1^{*}\right)$ satisfied. Condition $\left(\mathrm{D} 2^{\prime}\right)$ is met by the induction hypothesis and $\left(\mathrm{D}^{\prime}\right)$ by definition of $\mathscr{S}_{t, 1 / 2}$.

Terras [14] says that $\mathscr{F}_{n}^{\prime}$ has a "box shape" at infinity. For example, considering $n=2$, the portion of $\mathscr{F}_{2}^{\prime}$ with $y>1$ is a semi-infinite strip of width one. It can be seen fairly easily that $\mathscr{S}_{1,1 / 2}$ is the largest Siegel set contained in $\mathscr{F}_{n}^{\prime}$ and $\mathscr{S}_{4 / 3,1 / 2}$ is the smallest containing $\mathscr{F}_{n}{ }^{\prime}$. Again, this is well known in the case $n=2$, where

$$
\mathscr{S}_{t, 1 / 2}=\left\{z \in H:|x| \leq 1 / 2, y \geq \sqrt{t^{-1}}\right\} .
$$

It is also clear from that same picture that

$$
\mathscr{S}_{4 / 3,1 / 2} \subset \mathscr{F}_{2}^{\prime} \cup \mathscr{F}_{2}^{\prime}[\mathscr{S}]
$$

where $S=\left(\begin{array}{cc}0 & -1 \\ 1 & 0\end{array}\right)$. Roelcke [11] uses the fact that it takes just two copies of the fundamental domain to cover the smallest Siegel set containing the fundamental domain to obtain a lower bound for the eigenvalues of the Laplacian on $L^{2}(H / \operatorname{SL}(2, \mathbf{Z}))$. In this case, $|\lambda|>$ $3 \pi^{2} / 2$. Huntley [10] has extended these ideas to $L^{2}\left(\mathscr{S P P}_{3} / \Gamma_{3}\right)$ and shows that there $|\lambda|>3 \pi^{2} / 10$. To do this one needs to know that it takes 10 copies of the fundamental domain to cover $\mathscr{S}_{4 / 3,1 / 2}$ in $\mathscr{S P}_{3}$. From well known properties of the Siegel sets (see for example [2]) it is clear that the number is finite, but we need the exact number. This is related to the reduction algorithm for $\mathscr{S P P}_{3}$ discussed in [4] where it was necessary to form matrices $\gamma \in \Gamma_{3}$ having $a$ and $c$ as their first column for all the $a$ and $c$ necessary in (D1). However, there is an infinite number of choices for these matrices. For the application to Roelcke's method it is important to select these matrices more carefully.

Since we are using $\mathscr{F}_{3}^{\prime}$ here, (D1) gives the inequalities:

(i) $x_{12}^{2}+y_{1}^{2} \geq 1$,

(ii) $x_{13}^{2}+y_{1}^{2}\left(x_{23}^{2}+y_{2}^{2}\right) \geq 1$,

(iii) $\left(x_{12}+x_{13}\right)^{2}+y_{1}^{2}\left(\left(1+x_{23}\right)^{2}+y_{2}^{2}\right) \geq 1$,

(iv) $\left(1+x_{12}+x_{13}\right)^{2}+y_{1}^{2}\left(\left(1+x_{23}\right)^{2}+y_{2}^{2}\right) \geq 1$.

The difference in the inequalities here comes from the symmetry about $x_{12}=0$ which $\mathscr{F}_{3}^{\prime}$ has and $\mathscr{F}_{3}$ has not.

To get the smallest number of copies of $\mathscr{F}_{3}^{\prime}$ needed to cover 
$\mathscr{S}_{4 / 3,1 / 2}$, i.e., the number of $\gamma_{i}$ needed so that

$$
\mathscr{S}_{4 / 3,1 / 2} \subset \bigcup_{\gamma_{i}} \mathscr{F}_{3}{ }^{\prime}\left[\gamma_{i}\right]
$$

we need to make sure that for any $\gamma_{i}, Z \in \mathscr{F}_{3}^{\prime}\left[\gamma_{i}\right]$ satisfies $\left|x_{i j}\right| \leq 1 / 2$ for all $i, j$ where we use the Iwasawa decomposition (2). Take any $Z \in \mathscr{S}_{4 / 3,1 / 2}$ and use the reduction algorithm to move it to $\mathscr{F}_{3}^{\prime}$. (See [4] for the construction of a reduction algorithm, although we will need different matrices $S_{i}$ here.) First, if (using the decomposition (1)) $Z^{\prime} \notin \mathscr{F}_{2}^{\prime}$ then $Z\left[S^{\prime}\right]$ with

$$
S^{\prime}=\left(\begin{array}{ll}
1 & O \\
O & S
\end{array}\right)=\left(\begin{array}{lll}
1 & 0 & 0 \\
0 & 0 & 1 \\
0 & 1 & 0
\end{array}\right)
$$

has $Z^{\prime} \in \mathscr{F}_{2}^{\prime}$. At the same time this switches $x_{1}$ and $x_{2}$ where ${ }^{T} x=\left(x_{1}, x_{2}\right)$, but this is no problem since each satisfies $\left|x_{i}\right| \leq 1 / 2$. Thus, we may take $Z \in \mathscr{S}_{4 / 3,1 / 2}$ to satisfy conditions (D2') and $\left(\mathrm{D}^{\prime}\right)$ for $\mathscr{F}_{3}^{\prime}$, and we need to find $\gamma \in \Gamma_{3}^{\prime}$ so that $Z[\gamma] \in \mathscr{F}_{3}^{\prime}$. In [6] it is seen that for $Z[\gamma] \in \mathscr{F}_{3}^{\prime}$ we have for all $\gamma \in \Gamma_{3}^{\prime}$ :

$$
a_{1}(Z[\gamma]) \leq a_{1}(Z[\gamma][M])
$$

where $a_{1}(Z)$ denotes the upper left entry of $Z$ as before. If we take $M=\gamma^{-1}$ we have $\left(a w+{ }^{T} x c\right)^{2}+y^{3 / 2} Z^{\prime}[c] \leq 1$. So, we need only consider $\gamma$ with $a$ and $c$ satisfying this last inequality. Also based on results in [6] it can be shown that this inequalty can never be satisfed $\left(\bmod D_{3}\right)$ unless:

$$
\pm\left(\begin{array}{l}
a \\
c
\end{array}\right)=\left(\begin{array}{l}
1 \\
0 \\
0
\end{array}\right),\left(\begin{array}{l}
0 \\
1 \\
0
\end{array}\right),\left(\begin{array}{l}
0 \\
0 \\
1
\end{array}\right),\left(\begin{array}{l}
0 \\
1 \\
1
\end{array}\right),\left(\begin{array}{l}
1 \\
1 \\
1
\end{array}\right) \text {. }
$$

So we need to find matrices $S_{i}$ wth the first columns above. Experimentation showed that the right choices of $S_{i}$ are:

$$
\begin{aligned}
S_{1}=\left(\begin{array}{rrr}
0 & 1 & 0 \\
1 & 0 & 0 \\
0 & 0 & 1
\end{array}\right) & S_{2}=\left(\begin{array}{rrr}
0 & 0 & 1 \\
0 & 1 & 0
\end{array}\right), \\
S_{3}=\left(\begin{array}{rrr}
0 & 0 & 1 \\
1 & 1 & -1 \\
1 & 0 & 0
\end{array}\right), & S_{4}=\left(\begin{array}{rrr}
1 & 0 & 0 \\
1 & -1 & 0 \\
1 & 0 & -1
\end{array}\right),
\end{aligned}
$$

and $S_{5}=I$. What is meant by calling these the "right choices" is that there are infinitely many choices of matrices with the same first 
columns, but in each case $S_{i}$ is the only one with that first column to not affect $\left(\mathrm{D} 2^{\prime}\right)$ and $\left(\mathrm{D}^{\prime}\right)$. Also, each $S_{i}$ fixes the piece of the boundary of $\mathscr{F}_{n}^{\prime}$ from inequality (i) above. Note that for each $i$, $S_{i}^{2}=I$. We will say more on this shortly.

Returning to the problem at hand, since $\left(S^{\prime}\right)^{e} S_{i}, i=1,2,3,4,5$, $e=0$ or 1 move $Z$ into $\mathscr{F}_{3}{ }^{\prime}$, the inverses $S_{i}\left(S^{\prime}\right)^{e}$ are what we need to cover $\mathscr{S}_{4 / 3,1 / 2}$ with images of $\mathscr{F}_{3}{ }^{\prime}$, i.e.,

$$
\mathscr{S}_{4 / 3,1 / 2} \subset \bigcup \mathscr{F}_{3}^{\prime}\left[S_{i}\left(S^{\prime}\right)^{e}\right]
$$

To check that these are sufficient, recall that only matrices of the form $\left(S^{\prime}\right)^{e} S$ with $S$ having first column from the list above would move $Z \in \mathscr{S}_{4 / 3,1 / 2}$ into $\mathscr{F}_{3}{ }^{\prime}$. If we were to use a different $S$ from one of the $S_{i}, S=S_{i} g, g \in \mathrm{GL}(3, \mathbf{Z})$. Specifically, $S=S_{i}\left({ }_{O}^{1}{ }_{R}^{T} q\right)$, with $q \in \mathbf{Z}^{2}, R \in \mathrm{GL}(2, \mathbf{Z})$, in other words, $S \in P(1,2)$ where $\mathrm{P}(1,2)$ is the parabolic subgroup of $\mathrm{GL}(3, Z)$ consisting of matrices of this form. Now, if $Z^{\prime}=Z\left[\left(S^{\prime}\right)^{e} S_{i}\right] \in \mathscr{F}_{3}^{\prime}$, then $Z^{\prime}[g] \in \mathscr{F}_{3}^{\prime} \Rightarrow g=I$ as long as $Z^{\prime}$ is not on the boundary of $\mathscr{F}_{3}^{\prime}$. It is easily checked that they are all necessary. Thus, it takes 10 images of $\mathscr{F}_{3}^{\prime}$ to cover $\mathscr{S}_{4 / 3,1 / 2}$. This may be simplified and generalized to any $n$ by the following:

THEOREM 2. If $M_{i}$ are the minimal set such that

$$
\mathscr{S}_{4 / 3,1 / 2} \subset \bigcup \mathscr{F}_{n-1}^{\prime}\left[M_{i}\right] \text { in } \mathscr{S P P}_{n-1} \text {, }
$$

then in $\mathscr{S P}_{n}, \mathscr{S}_{4 / 3,1 / 2} \subset \cup \mathscr{F}_{n}^{\prime}\left[S_{j}\right]\left[M_{i}^{\prime}\right]$ where $M_{i}^{\prime}=\left(\begin{array}{ll}1 & O \\ O & M_{i}\end{array}\right)$ and the $S_{j}$ are those uniquely determined matrices $(\bmod P(1, n-1))$ with first columns given by the necessary and sufficient $a$ and $c$ in condition (D1) of Definition 1, which fix the corresponding pieces of the boundary of $\mathscr{F}_{n}{ }^{\prime}$. These satisfy $S_{j}^{2}=I$.

Most of this is clear from the preceding discussion, so we prove that we must have $S_{j}^{2}=I$. The Riemannian metric on $\mathscr{S P}_{n}$ is defined by:

$$
d s^{2}=\operatorname{Tr}\left(\left(d Y Y^{-1}\right)^{2}\right) .
$$

Let $Z_{0}$ be on the boundary fixed by $S_{j}$ but not on the other portions of the boundary, and let $Z_{1} \in \mathscr{F}_{n}^{\prime}$ (not on the boundary) be within a distance $\varepsilon$ of $Z_{0}$ where $\varepsilon$ is small enough so that no point on any other boundary piece is within $\varepsilon$ of $Z_{0}$. Then, since the map 
$Z \rightarrow Z[g]$ is continuous in $Z, Z_{1}\left[S_{j}\right]$ must be within $\varepsilon_{1}$ of $Z_{0}$, and $Z_{1}\left[S_{j}\right] \in \mathscr{F}_{n}^{\prime}\left[S_{j}\right]$. Similarly, $Z_{1}\left[S_{j}\right]\left[S_{j}\right]=Z_{1}\left[S_{j}^{2}\right]$ is within $\varepsilon_{2}$ of $Z_{0}$. By adjusting $\varepsilon$ we can make sure $\varepsilon_{2}$ is small enough so that no other boundary portion is within $\varepsilon_{2}$ of $Z_{0}$. Thus $Z_{1}\left[S_{J}^{2}\right] \in \mathscr{F}_{n}^{\prime}$. This gives $S_{j}^{2}=I$.

4. Compactification of $\mathscr{F}_{n}$. In this section we consider:

THEOREM 3. Let $\mathscr{F}_{n}{ }^{*}=\bigcup_{P} \mathscr{F}_{P}$ where if $P$ is a partition of $n$ written $\left(n_{1}, n_{2}, \ldots, n_{k}\right)$

$$
\mathscr{F}_{\left(n_{1}, \ldots, n_{k}\right)}=\mathscr{F}_{n_{1}} \times \cdots \times \mathscr{F}_{n_{k}}
$$

Then, with the topology defined below, $\mathscr{F}_{n}^{*}$ is a Satake compactification of $\mathscr{F}_{n}$, i.e. $\mathscr{F}_{n}^{*}$ is a compact Hausdorff space whose topology is induced by the closure of $\mathscr{F}_{n}$ and this topology induces a compactification of $\mathscr{S P P}_{n} / \Gamma_{n}$.

Here $\mathscr{F}_{1}$ is just $\{\infty\}$, a single point, as in the compactification of $\mathscr{F}_{2}, \mathscr{F}_{2}^{*}=\mathscr{F}_{2} \cup\{\infty\}$.

Recall that $a_{1}(Z)$ is defined to be the upper left entry of the matrix $Z$. Then for $\left(Z_{r}, Z_{s}\right) \in \mathscr{F}_{r} \times \mathscr{F}_{s}$ with $n=r+s$ and $U$ a neighborhood of $\left(Z_{r}, Z_{s}\right)$ in $\mathscr{F}_{r} \times \mathscr{F}_{s}$, define a neighborhood of $\left(Z_{r}, Z_{s}\right)$ in $\mathscr{F}_{n}{ }^{*}$ as follows: write $Z_{r}$ and $Z_{s}$ as

$$
\begin{aligned}
& Z_{r}=a_{1}\left(Z_{r}\right)\left(\begin{array}{lllll}
1 & & & & \\
& y_{1}^{2} & & & \\
& & \left(y_{1} y_{2}\right)^{2} & & \\
& & & \ddots & \\
& & & & \left(y_{1} y_{2} \ldots y_{r-1}\right)^{2}
\end{array}\right)\left[\begin{array}{lll}
1 & x_{i j} & \\
& \ddots & \\
& & 1
\end{array}\right] \\
& Z_{s}=a_{1}\left(Z_{s}\right)\left(\begin{array}{lllll}
1 & & & & \\
& y_{r+1}^{2} & & & \\
& & \left(y_{r+1} y_{r+2}\right)^{2} & & \\
& & & \ddots & \\
& & & & \left(y_{r+1} \ldots y_{n-1}\right)^{2}
\end{array}\right)\left[\begin{array}{ccc}
1 & x_{i j} & \\
& \ddots & \\
& & 1
\end{array}\right] \text {. }
\end{aligned}
$$

Then define $y$ by

$$
y^{n / 2}=\prod_{j=1}^{n-1} y_{j}^{n-j}
$$


and for $M>0$ let

$$
\begin{aligned}
& V(U, M)=\left\{Z \in \mathscr{S P P}_{n} \mid Z\right. \\
& =y^{-1}\left(\begin{array}{cc}
a_{1}\left(Z_{r}\right)^{-1} Z_{r} & O \\
O & a_{1}\left(Z_{S}\right)^{-1}\left(y_{1} \ldots y_{r}\right)^{2} Z_{s}
\end{array}\right)\left[\begin{array}{cc}
I_{r} & X \\
O & I_{s}
\end{array}\right] \\
& \text { with } y_{r}>M, Z_{r} \times Z_{s} \in U \text {, } \\
& \left.X=\left(x_{i j}\right) \text { with }\left|x_{i j}\right| \leq 1 / 2, x_{r 1} \geq 0\right\} \text {. }
\end{aligned}
$$

If $r$ or $s$ is 1 , we must take $Z_{r}$ or $Z_{s}$ to be 1 . Now define a neighborhood of $\left(Z_{r}, Z_{s}\right)$ in $\mathscr{F}_{n}^{*}$ by $V^{*}(U, M)=V(U, M) \cup U$. This definition can then be extended to define a neighborhood in $\mathscr{F}_{n}^{*}$ of $\left(Z_{1}, \ldots, Z_{k}\right) \in \mathscr{F}_{n_{1}} \times \cdots \times \mathscr{F}_{n_{k}}$. This sort of thing is usually called the Satake topology.

We need now show that for $M$ sufficiently large, $V(U, M) \subset \mathscr{F}_{n}$. To prove condition (D1),

$$
y Z\left[\begin{array}{l}
a \\
c
\end{array}\right]=\left(a+{ }^{T} x c\right)^{2}+Z_{1}[c] \geq 1,
$$

note that since $Z$ is a quadratic form, then if $c_{j}=0$ for $j \geq r$,

$$
Z\left[\begin{array}{l}
a \\
c
\end{array}\right]=Z_{r}[m]
$$

where $m \in \mathbf{Z}^{n-r}$ and ${ }^{T} m=\left(a, c_{1}, \ldots, c_{r-1}\right)$. But $Z_{r} \in \mathscr{F}_{r}$ so $y Z_{r}[m] \geq 1$. If $c_{j} \neq 0$ for some $j \geq r$, then we may apply Lemma 2 . We have:

$$
\left(a+{ }^{T} x c\right)^{2}+Z_{1}[c] \geq Z_{1}[c] \geq y_{1}^{2} \cdots y_{r}^{2} \geq(3 / 4)^{r-1} M^{2} .
$$

Thus, if we choose $M>(4 / 3)^{(r-1) / 2}$ we have $\left(a+{ }^{T} x c\right)^{2}+Z_{1}[c] \geq 1$. Now we must show that $Z^{\prime} \in \mathscr{F}_{n-1}$. Since $y^{\prime} Z^{\prime}=y_{1}^{-2} Z_{1}$ and so on, this is equivalent to showing that $y_{i}^{-2} Z_{i}[c] \geq 1$ for all $0 \neq c \in \mathbf{Z}^{n-i}$ and $i=1, \ldots, n-1$. Since

$$
y_{i}^{-2} Z_{i}[c] \geq Z_{i+1}\left[c^{\prime}\right]
$$

this follows immediately from Lemma 2 for $i=1, \ldots, r-1$ using the same arguments as above. For $i=r, Z^{\prime} \in \mathscr{F}_{n-1}$ follows from Lemma 1 , and from $Z_{s} \in \mathscr{F}_{s}$ for $i>r$. Condition (D3) clearly holds by the definition of $V(U, M)$.

To show compactness of $\mathscr{F}_{n}^{*}$ it will suffice to show that any sequence $\left\{Z^{(\nu)}\right\}$ in $\mathscr{F}_{n}$ has a limit point in $\mathscr{F}_{n}{ }^{*}$. Write the Iwasawa decomposition (2) of $Z^{(\nu)}$ with $y^{(\nu)}, y_{i}^{(\nu)}$ in place of the $y, y_{i}$. If 
all the sequences $\left\{y_{i}^{(\nu)}\right\}$ are bounded, then $Z^{(\nu)} \rightarrow Z \in \mathscr{F}_{n}$. If $\left\{y_{r}^{(\nu)}\right\}$ for some $r$ is not bounded, let $a_{r}$ be defined by

$$
a_{r}^{r / 2}=\prod_{j=1}^{r-1}\left(y_{j}^{(\nu)}\right)^{n-j}
$$

and write

$$
Z^{(\nu)}=\left(\begin{array}{cc}
\left(y^{(\nu)}\right)^{-1} a_{r} Z_{r}^{(\nu)} & O \\
O & \left(y^{(\nu)} a_{r}^{-1}\right)^{n_{1} / n_{2}} Z_{n-r}^{(\nu)}
\end{array}\right)\left[\begin{array}{cc}
I_{r} & X^{(\nu)} \\
O & I_{s}
\end{array}\right]
$$

Then $Z_{i}^{(\nu)} \in \mathscr{F}_{i}$ for $i=r$ or $n-r$ and if no other $\left\{y_{j}^{(\nu)}\right\}$ is unbounded, $Z_{i}^{(\nu)}$ converges to some $Z_{i} \in \mathscr{F}_{i}$. If another $\left\{y_{j}^{(\nu)}\right\}$ is unbounded we can repeat for $Z_{i}^{(\nu)}$ for whichever $i, r$ or $n-r$. Thus, we can establish the desired result inductively.

For some small $n$ we can write out $\mathscr{F}_{n}{ }^{*}$ :

$$
\mathscr{F}_{3}{ }^{*}=\mathscr{F}_{3} \cup \mathscr{F}_{2} \cup \mathscr{F}_{2} \cup \mathscr{F}_{1}
$$

where we write $\mathscr{F}_{2}$ instead of $\mathscr{F}_{2} \times \mathscr{F}_{1}$ since $\mathscr{F}_{1}$ is just one point. Note that this holds up very well with what we saw in $\S 2$. We also have

$$
\mathscr{F}_{4}{ }^{*}=\mathscr{F}_{4} \cup \mathscr{F}_{3} \cup \mathscr{F}_{2} \times \mathscr{F}_{2} \cup \mathscr{F}_{3} \cup \mathscr{F}_{2} \cup \mathscr{F}_{2} \cup \mathscr{F}_{2} \cup \mathscr{F}_{1}
$$

We can think of the first $\mathscr{F}_{2}$ as being a subspace of the first $\mathscr{F}_{3}$ as well as $\mathscr{F}_{2} \times \mathscr{F}_{2}$ in the Satake topology while the second $\mathscr{F}_{2}$ would be a subspace of each $\mathscr{F}_{3}$. This follows the containments of the appropriate parabolic subgroups..

If $\pi_{n}$ is the canonical projection of $\mathscr{S P}_{n}$ onto $\mathscr{S P P}_{n} / \Gamma_{n}$, then $\pi_{n}$ identifies $\mathscr{S P P}_{n} / \Gamma_{n}$ with $\mathscr{F}_{n}$. Using this, and letting $V_{n}=\mathscr{S P P}_{n} / \Gamma_{n}$, we get the Satake compactification $V_{n}^{*}=\bigcup_{P} V_{P}$ where if $P$ is the partition of $n$ into $\left(n_{1}, n_{2}, \ldots, n_{k}\right)$ or the parabolic subgroup $P\left(n_{1}, n_{2}, \ldots, n_{k}\right)$, then $V_{P}=V_{n_{1}} \times \cdots \times V_{n_{k}}$. Satake [13] actually defines several different compactifications of a quotient space (see also [16]); the one we have investigated here is the maximal Satake compactification. In [6] the compactification considered was not this maximal one. If we go all the way back to the beginning and restrict ourselves to the partial Iwasawa decompositions (1) we would have $\mathscr{F}_{n}{ }^{*}$ defined recursively by:

$$
\mathscr{F}_{n}{ }^{*}=\mathscr{F}_{n} \cup \mathscr{F}_{n-1}^{*}=\mathscr{F}_{n} \cup \mathscr{F}_{n-1} \cup \cdots \cup \mathscr{F}_{2} \cup \mathscr{F}_{1}
$$


with the topology defined accordingly by taking a neighborhood of $Z^{\prime} \in \mathscr{F}_{n-1}^{*}, U$, and defining its neighborhood in $\mathscr{F}_{n}^{*}$ to be:

$$
\begin{aligned}
\left\{Z \in \mathscr{S P}_{n} \mid Z=\left(\begin{array}{cc}
y^{-1} & O \\
O & y^{1 /(n-1)} Z^{\prime}
\end{array}\right)\left[\begin{array}{cc}
1 & T_{x} \\
O & I_{n-1}
\end{array}\right]\right. \\
\text { with } y>M, Z^{\prime} \in U,{ }^{T} x=\left(x_{1}, \ldots, x_{n-1}\right) \\
\left.\quad \text { with }\left|x_{j}\right| \leq 1 / 2, x_{1} \geq 0\right\} .
\end{aligned}
$$

Thus we have:

THEOREM 4. $\mathscr{F}_{n}^{*}=\mathscr{F}_{n} \cup \mathscr{F}_{n-1} \cup \cdots \cup \mathscr{F}_{2} \cup \mathscr{F}_{1}$ is a Satake compactification of $\mathscr{F}_{n}$. If $V_{n}=\mathscr{S P P}_{n} / \Gamma_{n}$ then $V_{n}^{*}=V_{n} \cup V_{n-1} \cup \cdots \cup V_{1} \cup V_{0}=$ $V_{n} \cup V_{n-1}^{*}$ is a Satake compactification of $V_{n}$.

This last compactification is analogous to Satake's original compactification of Siegel's quotient space [12].

If $\Gamma^{\prime}$ is any arithmetic subgroup of $G$, we can similarly obtain the compactifications of $V^{\prime}=\mathscr{S P}_{n} / \Gamma^{\prime}$. If $\mathscr{F}_{n}^{\prime}$ is the fundamental domain corresponding to $\mathscr{S P P}_{n} / \Gamma^{\prime}$ and $\left(\mathscr{F}_{n}^{\prime}\right)^{*}$ its compactification, Satake [13] shows that $\bigcup_{\gamma^{\prime} \in \Gamma^{\prime}}\left(\mathscr{F}_{n}^{\prime}\right)^{*}\left[\gamma^{\prime}\right]=\bigcup_{\gamma \in \Gamma_{n}} \mathscr{F}_{n}^{*}[\gamma]$, and that the topologies on these spaces are the same.

\section{REFERENCES}

[1] W. Baily and A. Borel, Compactification of arithmetic quotients of bounded symmetric domains, Ann. of Math., 84 (1966), 442-528.

[2] A. Borel, Introduction aux Groupes Arithmetiques, Hermann, Paris, 1969.

[3] J. Cassels, Rational Quadratic Forms, Academic Press, London, 1978.

[4] D. Gordon, D. Grenier, and A. Terras, Hecke operators and the fundamental domain for SL(3, Z), Math. Comp., 48 (1987), 159-178.

[5] D. Grenier, Fundamental Domains for $\mathscr{P}_{n} / \mathrm{GL}(n, \mathbf{Z})$ and Applications in Number Theory, Dissertation, UCSD, 1986.

[6] _ _ Fundamental domains for the general linear group, Pacific J. Math., 132 (1988), 293-317.

[7] _ An analogue of Siegel's $\phi$-operator for automorphic forms for $\operatorname{GL}(n, \mathbf{Z})$, Trans. Amer. Math. Soc., to appear.

[8] C. Hermite, Oeuvres I, Gauthier-Villars, Paris, 1905.

[9] J. Huntley, Multiplicity One Theorems for Automorphic Forms, Ph.D. Thesis, Stanford University, 1987.

[10] J. Huntley, correspondence.

[11] W. Roelcke, Über die Wellengleichung bei Grenzkreisgruppen erster Art, Sitzber. Akad. Heidelberg, Math-naturwiss., 1953/55.

[12] I. Satake, On the compactification of the Siegel space, J. Indian Math. Soc., 20 (1956), 259-281. 
[13] _ On compactifications of the quotient spaces for arithmetically defined discontinuous groups, Ann. of Math., 72 (1960), 555-580.

[14] A. Terras, Harmonic Analysis on Symmetric Spaces and Applications II, Springer-Verlag, New York, 1988.

[15] R. Zimmer, Ergodic Theory and Semisimple Groups, Birkhäuser, Boston, 1984.

[16] S. Zucker, $L^{p}$-cohomology and Satake compactifications, preprint.

Received May 20, 1991 and in revised form August 21, 1991.

THE JOHNS HOPKINS UNIVERSITY

BALTIMORE, MD 21218 


\section{CONTENTS}

G. D. Anderson, M. K. Vamanamurthy, and M. Vuorinen, Inequalities for quasi-

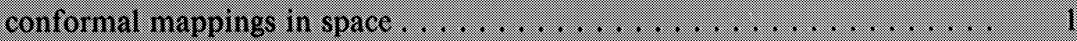

T. Bhattacharya, A nonexistence result for the $n$-Laplacian . . . . . . . . . . . . 19

J. A. Cima, K. Stroethoff, and K. Yale, Bourgain algebras on the unit disk . . . . . 27

J. A. Fridy and C. Orhan, Lacunary statistical convergence . . . . . . . . . . . . . . 43

D. Grenier, On the shape of fundamental domains in $\mathrm{GL}(n, \mathbf{R}) / \mathrm{O}(n) \ldots \ldots . . .53$

B. Jiang and J. Guo, Fixed points of surface diffeomorphisms . . . . . . . . . . . 67

P. Lejarraga, The moduli of rational Weierstrass fibrations over $\mathbf{P}^{\prime}$ : singularities 91

G. J. Martin, On discrete isometry groups of negative curvature . . . . . . . . . . 109

T. Nakashima, Adjoint linear systems on a surface of general type in positive characteristic . . . . . . . . . . . . . . . . . . . . . . . . . 129

B. Ralph, A homotopy transfer for finite group actions $\ldots \ldots \ldots \ldots \ldots \ldots \ldots$

Y. Rong, Maps between Seifert fibered spaces of infinite $\pi_{1} \ldots \ldots \ldots \ldots \ldots, 143$

J.-Y. Shi, Some numeric results on root systems . . . . . . . . . . . . 155

E. Spanier, Singular homology and cohomology with local coefficients and duality

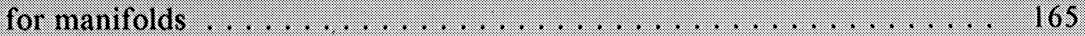




\section{PACIFIC JOURNAL OF MATHEMATICS}

Volume $160 \quad$ No. $1 \quad$ September 1993

Inequalities for quasiconformal mappings in space

GLEN DOUglas ANDERson, MaVina KRISHNa VAMANAMURThy and MATTI VUORINEN

A nonexistence result for the $n$-Laplacian

TILAK BHATTACHARYA

Bourgain algebras on the unit disk

Joseph A. Cima, Karel M. Stroethoff and Keith Yale

Lacunary statistical convergence

JOHN ALBERT FRIDY and CIHAN ORHAN

On the shape of fundamental domains in $\operatorname{GL}(n, \mathbf{R}) / \mathrm{O}(n)$

DOUGLAS MARTIN GRENIER

Fixed points of surface diffeomorphisms

BOJU JIANG and JIANHAN GUO

The moduli of rational Weierstrass fibrations over $\mathbf{P}^{1}$ : singularities

PABLO LEJARRAGA

On discrete isometry groups of negative curvature

GAVEN MARTIN

Adjoint linear systems on a surface of general type in positive characteristic

TOHRU NAKASHIMA

A homotopy transfer for finite group actions

WILLIAM J. RALPH

Maps between Seifert fibered spaces of infinite $\pi_{1}$

YONGWU RONG

Some numeric results on root systems

J. Y. SHI

Singular homology and cohomology with local coefficients and duality for manifolds

EDWIN SPANIER 